\title{
Nivel de conocimiento sobre enfermedades de transmisión sexual en jóvenes del Barrio Puzo, Distrito De Chilca 2019
}

\author{
Level of knowledge about sexually transmitted diseases in young people of the Barrio de Puzo, District of \\ Chilca 2019
}

Sara Palomino Hinojosa ${ }^{1, a}$, Edith Suasnabar Cueva ${ }^{1, b}$

\section{RESUMEN}

Objetivo: Determinar el nivel de conocimiento sobre las Enfermedades de Transmisión Sexual en jóvenes del Barrio de Puzo, Distrito de Chilca 2019.Material y métodos: La variable de estudio fue el nivel de conocimiento sobre las Enfermedades de Trasmisión Sexual, dentro de la metodología se trabajó con el método científico, pues es una secuencia lógica de procedimientos, siendo una investigación básica de nivel descriptivo, con una población y muestra censal de 150 jóvenes, que oscilan entre los 18 a 29 años con 11 meses y 29 días. Resultados: Del total de $150(100 \%)$ jóvenes encuestados. 94 (62.7\%) presentan un nivel de conocimiento bajo, 52 (34.7\%) presentan un nivel de conocimiento medio y $4(2.7 \%)$ un nivel de conocimiento alto. Siendo el mayor porcentaje del $62.7 \%$ equivalente a 94 jóvenes que presentan un nivel de conocimiento bajo sobre Enfermedades de Transmisión Sexual. Conclusiones: se determinó que el nivel de conocimiento sobre Enfermedades de Transmisión Sexual en jóvenes del Barrio de Puzo, Distrito de Chilca 2019, es bajo con 62.7\% equivalente a 94 jóvenes.

PALABRAS CLAVE: Nivel de conocimiento, enfermedades de transmisión sexual, jóvenes.

\section{SUMMARY}

Objective: To determine the level of knowledge about sexually transmitted diseases in young people of the Barrio de Puzo, District of Chilca 2019. Material and Methods: The study variable is the level of knowledge about sexually transmitted diseases, within the methodology we worked with The scientific method, as it is a logical sequence of procedures, being a basic research of descriptive level, with a population and census sample of 150 young people, ranging between 18 to 29 years with 11 months and 29 days. Results: From $150(100 \%)$ young people surveyed. 94 $(62.7 \%)$ have a low level of knowledge, $52(34.7 \%)$ have a medium level of knowledge and $4(2.7 \%)$ have a high level of knowledge. The highest percentage of $62.7 \%$ is equivalent to 94 young people who have a low level of knowledge about sexually transmitted diseases. Conclusions: It was determined that the level of knowledge about sexually transmitted diseases in young people of the Barrio de Puzo, District of Chilca 2019. It is low with 62.7\% equivalent to 94 young people.

KEYWORDS: Level of knowledge, sexually transmitted diseases, youth.

\footnotetext{
Facultad de Ciencias de la Salud Escuela Profesional de Enfermería, Universidad Privada De Huancayo Franklin Roosevelt. Huancayo, Perú.

a Bachiller; ${ }^{\mathrm{b}}$ Licenciada, Docente.
} 


\section{INTRODUCCIÓN}

Según la Organización Mundial de la Salud (OMS) las Enfermedades de Trasmisión Sexual (ETS) son causadas por más de 30 bacterias, virus y parasitosis diferentes y se propagan predominantemente por contacto sexual (1).

Desde la aparición del Virus Inmunodeficiencia Humana y el Síndrome de Inmunodeficiencia Adquirida (VIH/SIDA) a nivel mundial han pasado más de 25 años y en el Perú más de 23 años. Es la enfermedad que ha ocasionado más muertes a nivel mundial, más de 60 millones de personas han sido afectadas con este mal y alrededor de 15 millones han muerto (2).

En el Perú según la dirección general de epidemiologia, se presentaron desde el año 1983 hasta octubre de 2017 unos 40,551 casos de personas con SIDA y 109263 casos de VIH registrados y cada día mueren entre 5 o 6 mujeres a causa de algún tipo de cáncer generado por Virus del Papiloma Humano (VPH), según la liga contra el cáncer al año registran 4,043 nuevos casos y 1.836 mujeres fallecen a causa de este enfermedad toda evidencia científica actual que el $99 \%$ de los casos de cáncer cuello uterino son a causa del VPH también se registraron 785 casos de sífilis en el Perú (3).

Las ETS constituyen un grave problema de salud pública en la mayor parte de los países, las cuales afectan principalmente a la población de jóvenes los que se constituyen como la población más vulnerable a contraer una Enfermedad de

Transmisión Sexual, siendo un indicador de riesgo el comportamiento sexual de los mismos que los impulsan a tener relaciones sexuales tempranamente (4).

El presente trabajo tuvo como objetivo determinar el nivel de conocimiento sobre enfermedades de transmisión sexual en jóvenes del Barrio de Puzo, Distrito de Chilca 2019.

\section{MATERIAL Y METODOS}

El método general utilizado en la presente investigación fue el método científico; es una secuencia lógica de procedimientos, con un alto grado de precisión y objetividad orientados a comprender y explicar rigurosamente los hechos por medio de la razón a fin de lograr su veracidad (5), y el método especifico fue descriptivo, consiste en un conjunto de procedimientos que nos permitió señalar las características en forma detallada y ordenada del problema de estudio.

La investigación fue básica, consiste en descubrir nuevos conocimientos mediante la descripción del fenómeno de estudio, es decir mediante la recopilación de información para enriquecer el conocimiento teórico científico; al respecto Bernal M. sostiene que este tipo de investigación nos lleva a la búsqueda de nuevos conocimientos y recoge información de la realidad para enriquecer el conocimiento teórico científico, y que nos orienta al descubrimiento de principios y leyes, por otro lado busca el progreso científico (5).

El presente trabajo tuvo el nivel de investigación descriptivo, el cual consiste en describir metódica y sistemáticamente las características del problema y de corte transversal (5).

En este trabajo de investigación se empleó el diseño descriptivo simple no experimental, según Montero y De La Cruz, el diseño descriptivo simple, pertenece al grupo de no experimental y permite señalar la manera de cómo se recogerán los datos de la muestra de estudio en un momento determinado (5).

La población de estudio es de 150 jóvenes que asistes al centro eclesiásticos, que se encuentran entre las edades de 18 a 29 años 11 meses y 29 días del Barrio Puzo, Distrito de Chilca.

La muestra utilizada fue la muestra censal, es aquella donde todas las unidades de investigación son consideradas como muestra (6).

\section{Criterios de inclusión}

Todos los jóvenes que se encuentran entre las edades de 18 a 29 años 11 meses y 29 días del Barrio Puzo, Distrito de Chilca.

Todos los jóvenes que aceptan el consentimiento informado.

\section{Criterios de exclusión}

Todos los jóvenes que no se encuentran entre las edades de 18 a 29 años 11 meses y 29 días del Barrio Puzo, Distrito de Chilca.

Todos los jóvenes que no aceptan el consentimiento informado. 
Todos los jóvenes que no son del Barrio Puzo, Distrito de Chilca.

La técnica utilizada fue La encuesta, consiste en recopilar información sobre una parte de la población denominada muestra, la información recogida podrá emplearse para un análisis cuantitativo con el fin de identificar y conocer la magnitud de los problemas que se suponen o se conocen en forma parcial o imprecisa (5).

El instrumento utilizado por medio del cual se obtuvo la información de nuestra muestra sobre el nivel de conocimiento sobre Enfermedades de Trasmisión Sexual en los Jóvenes del Barrio de Puzo, Distrito de Chilca, fue un cuestionario con 38 preguntas elaborado por la autora fue validado por juicio de expertos, el instrumento a pasado por la prueba de confiabilidad por alfa cronbach el resultado es 0.808 por lo tanto es altamente confiable, es un instrumento de investigación que contienen preguntas debidamente estructuradas con la finalidad de obtener información de un grupo de personas (5).

\section{RESULTADOS}

En la tabla 1 se observa del total de $150(100 \%)$ jóvenes encuestados. $94(62.7 \%)$ presentó un nivel de conocimiento bajo, $52(34.7 \%)$ presentó un nivel de conocimiento medio y $4(2.7 \%)$ un nivel de conocimiento alto. Siendo el mayor porcentaje del $62.7 \%$ equivalente a 94 jóvenes que presentó un nivel de conocimiento bajo sobre Enfermedades de Transmisión Sexual.

En la tabla 2 se observa del total de 150 (100\%) jóvenes encuestados. 113(75.3\%) presento un nivel de conocimiento bajo, 37(24.7\%) presento un nivel de conocimiento medio y $0(0 \%)$ un nivel de conocimiento alto. Siendo el mayor porcentaje del $75.3 \%$ equivalente a 113 jóvenes que presentan un nivel de conocimiento bajo sobre Enfermedades de Transmisión Sexual Sífilis.

En la tabla 3 se observa del total de $150(100 \%)$ jóvenes encuestados. 114(76\%) presento un nivel de conocimiento bajo, $30(20.0 \%)$ presento un

Tabla 1.Nivel de conocimiento sobre enfermedades de transmisión sexual en jóvenes del Barrio Puzo, Distrito de Chilca 2019.

\begin{tabular}{llcc}
\hline Nivel de Conocimiento & Categorías & Frecuencia & Porcentaje \\
\hline \multirow{4}{*}{ Valido } & Alto & 94 & 62.7 \\
& Medio & 52 & 34.7 \\
& Bajo & 4 & 2.7 \\
& Total & 150 & 100 \\
\hline
\end{tabular}

Tabla 2.Nivel de conocimiento sobre la sifilis en jóvenes del Barrio Puzo, Distrito de Chilca 2019.

\begin{tabular}{llcc}
\hline Nivel de Conocimiento & Categorías & Frecuencia & Porcentaje \\
\hline \multirow{3}{*}{ Valido } & Alto & 113 & 75.3 \\
& Medio & 37 & 24.7 \\
& Bajo & 0 & 0 \\
& Total & 150 & 100 \\
\hline
\end{tabular}

Tabla 3.Nivel de conocimiento sobre el virus del papiloma humano en jóvenes del Barrio Puzo, Distrito de Chilca 2019.

\begin{tabular}{llcc}
\hline Nivel de Conocimiento & Categorías & Frecuencia & Porcentaje \\
\hline \multirow{4}{*}{ Valido } & Alto & 114 & 76 \\
& Medio & 30 & 20 \\
& Bajo & 6 & 4 \\
& Total & 150 & 100 \\
\hline
\end{tabular}


Tabla 4. Nivel de conocimiento sobre el VIH-SIDA en jóvenes del Barrio Puzo, Distrito de Chilca 2019.

\begin{tabular}{llcc}
\hline Nivel de Conocimiento & Categorías & Frecuencia & Porcentaje \\
\hline \multirow{4}{*}{ Valido } & Alto & 84 & 56 \\
& Medio & 58 & 38.7 \\
& Bajo & 8 & 5.3 \\
& Total & 150 & 100 \\
\hline
\end{tabular}

nivel de conocimiento medio y $6(4.0 \%)$ un nivel de conocimiento alto. Siendo el mayor porcentaje del $76 \%$ equivalente a 114 jóvenes que presentan un nivel de conocimiento bajo sobre Enfermedades de Transmisión Sexual por Virus del Papiloma Humano. En la tabla 4 se observa del total de $150(100 \%)$ jóvenes encuestados. $84(56 \%)$ presento un nivel de conocimiento bajo, $58(38.7 \%)$ presento un nivel de conocimiento medio y $8(5.3 \%)$ un nivel de conocimiento alto. Siendo el mayor porcentaje del $56 \%$ equivalente a 84 jóvenes que presentan un nivel de conocimiento bajo sobre Enfermedades de Transmisión Sexual VIH-SIDA.

\section{DISCUSIÓN}

De los resultados encontrados del objetivo general: determinar el nivel de conocimiento sobre enfermedades de transmisión sexual en jóvenes del Barrio Puzo, Distrito de Chilca 2019. En la tabla 1 se observa del total de 150 (100\%) jóvenes encuestados. $94(62.7 \%)$ presento un nivel de conocimiento bajo, $52(34.7 \%)$ presento un nivel de conocimiento medio y 4(2.7\%) un nivel de conocimiento alto. Siendo el mayor porcentaje del $62.7 \%$ equivalente a 94 jóvenes que presentan un nivel de conocimiento bajo sobre enfermedades de transmisión sexual, los conocimientos que tienen los adolescentes respecto a las Enfermedades de Transmisión Sexual son escasos (4).

Asimismo, en la investigación de Martinez y Muñoz señalan que el $72 \%$ de la población respondió correctamente el cuestionario, sin embargo, no hay suficiente conocimiento sobre mecanismos de transmisión y características generales de otras ITS. Lo cual conlleva a la posibilidad de realizar prácticas de riesgo para la adquisición de una ITS (7).

De esta manera podemos mencionar que, los jóvenes pueden conocer el tema de ETS, pero ese conocimiento es bajo, notamos a través de nuestro estudio que solo $2.7 \%$ de nuestra población tiene el conocimiento alto en temas generales sobre ETS.
Según la Organización de las Naciones Unidas (ONUSIDA), son causadas por agentes bacterianos, protozoarios, tricomoniasis y escherichia coli se curan con antibióticos y agentes quimioterapéuticos apropiados desde más de 40 años, no obstante, estas ETS siguen construyendo un problema de salud pública (8).

De los resultados encontrados en el objetivo específico 1: Identificar el nivel de conocimiento sobre enfermedad de trasmisión sexual Sífilis en jóvenes del Barrio Puzo, Distrito de Chilca 2019. En la tabla 2 se observa del total de 150 (100\%) jóvenes encuestados. $113(75.3 \%)$ presento un nivel de conocimiento bajo, $37(24.7 \%)$ presento un nivel de conocimiento medio y $0(0 \%)$ un nivel de conocimiento alto. Siendo el mayor porcentaje del $75.3 \%$ equivalente a 113 jóvenes que presentan un nivel de conocimiento bajo sobre enfermedades de transmisión sexual sífilis. Este resultado difiere con el estudio de Vásquez E. en su trabajo de investigación titulado "Conocimientos y prácticas sobre la prevención de infecciones de transmisión sexual en los estudiantes de la unidad educativa general Córdova del distrito educativo 02 circuito c05.06 en el periodo julio-noviembre 2014. Concluyendo que el $91.5 \%$ de estudiantes conocen sobre las ITS, información obtenida a través de la televisión y con alguno de sus progenitores.

Si bien nuestro estudio difiere con el estudio de Vásquez E. puesto que no hay ni una sola persona de nuestra muestra que presente conocimiento alto sobre sífilis (10), esto es preocupante para el personal de salud, así como lo debería ser para las autoridades comunales, locales y provinciales que tienen una labor importante que realizar en materia de salud sexual. Pues se trata de jóvenes que en pleno desarrollo tecnológico y acceso a la información, sea bajo y medio su conocimientos sobre sifilis. A comparación de los estudiantes de la unidad educativa general Córdova del distrito educativo 02 circuito c05.06 que la información obtenida es a través de la televisión y sus progenitores. 
En la tabla 3 se observó que del total de 150 $(100 \%)$ jóvenes encuestados. 114(76\%) presento un nivel de conocimiento bajo, $30(20.0 \%)$ presento un nivel de conocimiento medio y $6(4.0 \%)$ un nivel de conocimiento alto. Siendo el mayor porcentaje del $76 \%$ equivalente a 114 jóvenes que presentan un nivel de conocimiento bajo sobre enfermedades de transmisión sexual por virus del papiloma humano. Este resultado es similar al estudio de Jaramillo concluyendo que $38,59 \%$ es el Virus de Papiloma Humana . Asimismo, en respaldo por la OMS el Virus del Papiloma Humano (VHP) causa cáncer de cuello de útero, ocupa el cuarto lugar entre los tipos más comunes de cáncer que afectan, con un número estimado de 266000 muertes y unos 528000 nuevos casos en el 2012. La gran mayoría (alrededor del 85 $\%)$ de esas muertes se produjeron en las regiones menos desarrolladas, donde es el causante de casi el $12 \%$ de todos los canceres femeninos. 18; estos datos permiten saber que, al ser una ETS que no tiene cura además, genera cáncer de cuello uterino y solo el $4.0 \%$ de mi muestra de estudio tiene conocimiento de esto; y debería ser un aspecto de gran interés en el campo de la salud.

En la tabla 4 se observó del total de 150 (100\%) jóvenes encuestados. $84(56 \%)$ presento un nivel de conocimiento bajo, $58(38.7 \%)$ presento un nivel de conocimiento medio y $8(5.3 \%)$ un nivel de conocimiento alto. Siendo el mayor porcentaje del $56 \%$ equivalente a 84 jóvenes que presentan un nivel de conocimiento bajo sobre enfermedades de transmisión sexual VIH-SIDA. Este resultado difiere con el estudio de Choccare quien concluye que los jóvenes tienen un nivel medio de conocimientos sobre las infecciones de transmisión sexual-VIH/SIDA (12). Igual resultado presenta el estudio de Bravo et al, quienes concluyen encontraron que para el nivel de conocimiento sobre trasmisión de VIH/SIDA el 49,6\% de estudiantes varones posee conocimiento medio (13).

Estos resultados demuestran que, a pesar de ser un tema bastante tocado en colegios, universidades, campañas de salud de parte de ONG, de centros de salud y otras instituciones; los jóvenes del barrio Puzo del distrito de chilca, tienen el nivel de conocimiento bajo con un $56 \%$ pero a la vez alentador en el sentido que, casi la otra mitad con $44 \%$ tienen el nivel alto y medio. Pero aún hay una gran diferencia con nuestros antecedentes que tienen porcentaje alto en nivel de conocimiento, por lo que, como personal de salud estamos en el compromiso y debes de contribuir de fomentar el conocimiento sobre estos temas de importancia, siempre de la mano con nuestras autoridades

\section{Limitaciónes de la investigación}

a) Limitación internas: Por el diseño del presente estudio los resultados obtenidos serán válidos solo para la población de estudio en este caso para los jóvenes del Barrio Puzo, distrito de Chilca.

b) Limitación externas: Para el uso del instrumento de recolección de datos no se contaba con la plena predisposición de los jóvenes del Barrio Puzo, distrito de Chilca, esto fue superado.

\section{CONCLUSIONES}

Se determinó que el nivel de conocimiento sobre Enfermedades de Transmisión Sexual en jóvenes del Barrio Puzo, Distrito de Chilca 2019, es bajo con $62.7 \%$ equivalente a 94 jóvenes.

Se identificó que el nivel de conocimiento sobre la Enfermedad de Trasmisión Sexual de Sífilis en los jóvenes del Barrio Puzo, Distrito de Chilca 2019, es bajo con $75.3 \%$ equivalente a 113 jóvenes.

Se identificó que el nivel de conocimiento sobre Enfermedades de Trasmisión Sexual por Virus del Papiloma Humano en los jóvenes del Barrio Puzo, Distrito de Chilca 2019, es bajo con $76 \%$ equivalente a 114 jóvenes.

Se identificó que el nivel de conocimiento sobre Enfermedad de Trasmisión Sexual VIH- SIDA en los jóvenes del Barrio Puzo, Distrito de Chilca 2019, es bajo con el $56 \%$ equivalente a 84 jóvenes.

Agradecimiento: A los jóvenes del Barrio Puzo, Distrito de Chilca, que desinteresadamente me apoyaron en la realización de este trabajo de investigación.

\section{Correspondencia}

Edith Suasnabar Cueva

Av. Giráldez 542, Huancayo 12001. Junin, Perú

\section{REFERENCIAS BIBLIOGRAFICAS}

1. Organización Mundial de la Salud. Infecciones de Trasmisión Sexual. Ginebra: Organización Mundial de la Salud; 2016. (Fecha de acceso 02 de marzo 
del 2019) disponible en: https://www.who.int/ topics/sexually_transmitted_infections/es/

2. Gonzales I. Síndrome de Inmunodeficiencia Adquirida: desarrollo histórico e importancia del conocimiento para su prevención. Rev Cubana Med Gen Integr. 2015; 31 (1):0-0. (Fecha de acceso 02 de marzo del 2019) disponible en:http://scielo. sld.cu/scielo.php?script=sci_arttext\&pid $=$ S0864-21252015000100013

3. Ministerio de Salud. Boletin Epidemiologico del Perú. Lima: Ministerio de Salud; 2018. (Fecha de acceso 02 de marzo del 2019) Disponible en: https://www.dge.gob.pe/portal/docs/vigilancia/ boletines/2018/33.pdf

4. Mercados2. Las enfermedades de transmisión sexual están aumentando entre los jóvenes. Lima: Mercados2; 2017. (Fecha de acceso 02 de marzo del 2019) Disponible en: https:/www.merca2.es/ enfermedades-transmision-sexual-aumentando/

5. Montero I, De La Cruz M. Metodología de la investigación científica. Huancayo: Editorial Graficorp; 2016

6. Ramírez P. Como realizar un proyecto de investigación - Caracas; 2004

7. Martinez C, Muñoz L. Nivel de conocimiento sobre infecciones de transmisión sexual en estudiantes de dos facultades de la universidad de ciencias aplicadas y ambientales a través de la escala sobre el VIH y otras infecciones de transmisión sexual (ECI) modificada. Bbogota: Universidad de Ciencias Aolicadas y Ambientales; 2016. (Fecha de acceso 02 de marzo del 2019) Disponible en: https:// repository.udca.edu.co/bitstream/11158/752/1/ Nivel $\% 20$ de $\% 20$ co nocimiento $\% 20$ sobre $\% 20$ ITS $\% 20$ en $\% 20$ dos $\% 20$ facultades $\% 20 d e \% 201 \mathrm{a} \% 20$ U.D.C.A.pdf

8. Augusto V.La teoría del conocimiento en investigación científica una visión actual. Anales de la Facultad de Medicina. 2009;70(3):217-224. (Fecha de acceso 02 de marzo del 2019) Disponible en: http:// www.redalyc.org/articulo.oa? $\mathrm{id}=37912410011$
9. Organización Mundial de la Salud. Infección de transmisión sexual. Ginebra Organización Mundial de la Salud; 2016.1 (Fecha de acceso 02 de marzo del 2019) Disponible en: https://www.who.int/ topics/sexually_transmitted_infections/es/

10. Vásquez E. Conocimientos y Practicas sobre la prevención de infecciones de transmisión sexual en los estudiantes de la unidad educativa general Córdova del distrito educativo 02 circuito c05.06 en el periodo julio - noviembre 2014. Tesis de Grado. Ambato: Universidad Tecnica de Ambato; 2015. (Fechadeacceso 02 de marzo del 2019) Disponible en: http://repo.uta.edu.ec/bitstream/123456789/9432/ $1 /$ tesis $\% 20$ erika $\% 20$ fernanda $\% 20$ vasquez $\% 20$ cherrez.pdf

11. Jaramillo G. Enfermedades de trasmisión sexual en adolescentes del colegio nacional 26 de noviembre Zaruma ECUADOR 2015" (Fecha de acceso 02 de marzo del 2019). Loja: Universidad Nacional de Loja; 2015. Disponible en: http://dspace.unl. edu.ec/jspui/bitstream/123456789/12160/1/TESIS. pdf

12. Choccare S. Nivel de conocimiento sobre las infecciones de transmisión sexual -VIH/SIDA y sus medidas preventivas en los estudiantes de enfermería de la UNMSM,2007: Lima, Perú: Universidad Nacional Mayor de San Marcos; 2008. (Fecha de acceso 02 de marzo del 2019) Disponible en: http://cybertesis.unmsm.edu.pee/bitstream/ handle/cybertesis/521/chocca re_sc.pdf;jsessionid $=0$ FF8056467B896398E94894055E41EBE?sequenc $\mathrm{e}=1$

13. Bravo P, Calle M, Lázaro L. Conocimientos sobre transmisión de VIH y actitudes sobre prácticas sexuales riesgosas en varones de una institución educativa de Nazca -Ica 2017. Lima, Peru: Universidad Peruana Cayetano Heredia; 2018. (Fecha de acceso 02 de marzo del 2019) Disponible en: http://repositorio.upch.edu.pe/bitstream/handle/ upch/3694/Conocimiento BravoMolina Patricia. pdf? sequence $=1$ \&isAllowed $=\mathrm{y}$

Recibido: $14 / 01 / 2020$

Aceptado: 16/06/2020 